\title{
Mathematical Modeling of Boundary Layer Flow over a Moving Thin Needle with Variable Heat Flux
}

\author{
SYAKILA AHMAD ${ }^{1}$ \\ NORIHAN MD. ARIFIN ${ }^{2}$ \\ Universiti Putra Malaysia \\ Institute for Mathematical Research \\ 43400 UPM Serdang, Selangor \\ MALAYSIA
}

\author{
ROSLINDA NAZAR \\ Universiti Kebangsaan Malaysia \\ School of Mathematical Sciences \\ 43600 UKM Bangi, Selangor \\ MALAYSIA
}

\author{
IOAN POP \\ University of Cluj \\ Faculty of Mathematics \\ CP 253, R-3400 Cluj \\ ROMANIA
}

Abstract: The problem of steady laminar forced convection boundary layer flow of an incompressible viscous fluid over a moving thin needle with variable heat flux is considered. The governing boundary layer equations are first transformed into non-dimensional forms. These equations are then transformed into similarity equations using the similarity variables, which are solved numerically using an implicit finite-difference scheme known as the Keller-box method. The solutions are obtained for a blunt-nosed needle $(m=0)$. Numerical computations are carried out for various values of the dimensionless parameters of the problem, which include the Prandtl number $\mathrm{Pr}$ and the parameter $a$ representing the needle size. It has been found that the wall temperature are significantly influenced by both parameters $a$ and Prandtl number Pr. However, the Prandtl number has no effect on the flow characteristics due to the decoupled boundary layer equations.

Key-Words: Boundary layer flow, Moving thin needle, Variable heat flux

\section{Introduction}

Thin needle is a body of revolution whose diameter is of the same order as the velocity or thermal boundary layers that it develops. By appropriately varying the radius of the needle, the partial differential boundary layer equations admit similarity solutions, which are more revealing than the direct numerical integration of the partial differential equations. As it is well-known, an important aspect of experimental studies for the flow and heat transfer characteristics is the measurements of velocity and temperature profiles of the flow field. The probe of the measuring devices, such as a hot wire anemometer or shielded thermocouple, is often a very thin wire or needle. Meanwhile, boundary layer behavior over moving solid surface is an important type of flow occurring in a number of engineering processes. Aerodynamic extrusion of plastic sheet, cooling of an infinite metallic plate in a cooling bath, the boundary layer along a liquid film in condensation processes and a polymer sheet or filament extruded continuously from a dye, or a long thread travelling between a feed roll and a wind-up roll, are examples of practical applications of continuous surfaces (see $[1,2])$. From an industrial point of view, the wall shear stress distribution is perhaps the most important parameter in this type of flow because it directly determines the driving force (or torque) required to withdraw the surface (see [3]). Therefore, the detailed analysis of the flow over such moving slender needleshaped bodies is of considerable practical interest.

The problems of forced, free and mixed convection boundary layer flows over thin needles have been investigated by many researchers. We mention here Chen and Smith [4], Narain and Uberoi [5, 6], Chen [7], Lee et al. [8] and Ahmad et al. [9] have studied various aspects of this problem. Wang [10] has studied the problem of mixed convection boundary layer flow on a vertical adiabatic thin needle with a concentrated heat source at the tip of the needle. This situation may be applied, for example, to a stick burning at the bottom end. Agarwal et al. [11] have investigated numerically the momentum and thermal boundary layers for power-law fluids over a thin needle under wide ranges of kinematic and physical conditions. We also notice to this end that Gorla $[12,13,14]$ has studied the boundary layer flow in the vicinity of an axisymmetric stagnation point on a circular cylinder placed in a Newtonian or in a micropolar fluid.

All studies mentioned above on forced, free or mixed convection boundary layer flows over thin needles refer to fixed needles immersed in a viscous and incompressible fluid. However, the solutions for 
mixed convection boundary layer flow past a vertical moving thin needle in a quiescent fluid with variable heat flux have been reported recently by Ahmad et al. [15]. The aim of this present paper is to study the problem of steady forced convection boundary layer flow over a moving thin needle with variable heat flux in a quiescent fluid. It should also be mentioned that due to entrainment of the ambient fluid, this flow situation represents an intrinsically different class of boundary layer flows, which have substantially different type of solutions as compared to the case of a static needle. By the similarity transformation, the partial differential equations governing the flow and temperature fields are reduced to ordinary differential equations, which are solved numerically using an implicit finite-difference scheme called the Keller-box method. The influences of the needle size and the Prandtl number on the flow and heat transfer characteristics are presented in graphical form.

\section{Mathematical Formulation}

Consider a steady laminar boundary layer flow of an incompressible viscous fluid over a moving thin needle in a bulk fluid at a constant temperature $T_{\infty}$. Figure 1 shows the slender paraboloid needle whose radius is described by $\bar{r}=\bar{R}(\bar{x})$, where $\bar{x}$ and $\bar{r}$ are the axial and radial coordinates, respectively, with the $\bar{x}-$ axis measured from the needle leading edge. The needle is considered thin when its thickness does not exceed that of the boundary layer over it. Under this assumption, the effect of transverse curvature is of importance, but the pressure variation along the surface due to the presence of the needle can be neglected (see [16]). It is assumed that the needle moves horizontally with the velocity $\bar{U}(\bar{x})$ and is subjected to a variable surface heat flux $\bar{q}_{w}(\bar{x})$. Under the boundary layer approximations, the basic boundary layer equations written in cylindrical coordinates are

$$
\begin{aligned}
\frac{\partial}{\partial \bar{x}}(\bar{r} \bar{u})+\frac{\partial}{\partial \bar{r}}(\bar{r} \bar{v}) & =0 \\
\bar{u} \frac{\partial \bar{u}}{\partial \bar{x}}+\bar{v} \frac{\partial \bar{u}}{\partial \bar{r}} & =\frac{\nu}{\bar{r}} \frac{\partial}{\partial \bar{r}}\left(\bar{r} \frac{\partial \bar{u}}{\partial \bar{r}}\right) \\
\bar{u} \frac{\partial \bar{T}}{\partial \bar{x}}+\bar{v} \frac{\partial \bar{T}}{\partial \bar{r}} & =\frac{\alpha}{\bar{r}} \frac{\partial}{\partial \bar{r}}\left(\bar{r} \frac{\partial \bar{T}}{\partial \bar{r}}\right)
\end{aligned}
$$

where $\bar{u}$ and $\bar{v}$ are the velocity components along the $\bar{x}-$ and $\bar{r}$ - axes, respectively, $\bar{T}$ is the local fluid temperature, $\nu$ is the kinematic viscosity and $\alpha$ is the constant thermal diffusivity of the fluid. We assume that the boundary conditions of Eqs. (1) - (3) are

$\bar{v}=0, \bar{u}=\bar{U}(\bar{x}), \frac{\partial \bar{T}}{\partial \bar{r}}=-\frac{\bar{q}_{w}(\bar{x})}{k}$ at $\bar{r}=\bar{R}(\bar{x})$

$$
\bar{u} \rightarrow 0, \bar{T} \rightarrow T_{\infty} \text { as } \bar{r} \rightarrow \infty
$$

where $\bar{R}(\bar{x})$ prescribes the surface shape of the axisymmetric body.

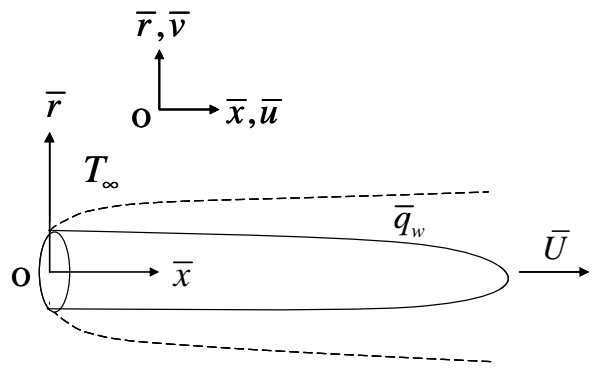

Figure 1: Physical model and coordinate system

We introduce now the following non-dimensional variables:

$$
\begin{array}{r}
x=\frac{\bar{x}}{L}, r=\operatorname{Re}^{1 / 2}\left(\frac{\bar{r}}{L}\right), u=\frac{\bar{u}}{U_{0}}, \\
v=\operatorname{Re}^{1 / 2}\left(\frac{\bar{v}}{U_{0}}\right), U(x)=\frac{\bar{U}(\bar{x})}{U_{0}}, \\
R(x)=R e^{1 / 2}\left(\frac{\bar{R}(\bar{x})}{L}\right), q_{w}(x)=\frac{\bar{q}_{w}(\bar{x})}{q_{0}}, \\
T=\frac{k R e^{1 / 2}\left(\bar{T}-T_{\infty}\right)}{q_{0} L},
\end{array}
$$

where $L$ is a characteristic length of the needle, $U_{0}$ is the characteristic velocity, $q_{0}$ is the characteristic heat flux and $R e=U_{0} L / \nu$ is the Reynolds number. Substituting (5) into Eqs. (1) - (3), we get

$$
\begin{aligned}
\frac{\partial}{\partial x}(r u)+\frac{\partial}{\partial r}(r v) & =0 \\
u \frac{\partial u}{\partial x}+v \frac{\partial u}{\partial r} & =\frac{1}{r} \frac{\partial}{\partial r}\left(r \frac{\partial u}{\partial r}\right) \\
u \frac{\partial T}{\partial x}+v \frac{\partial T}{\partial r} & =\frac{1}{\operatorname{Pr}} \frac{1}{r} \frac{\partial}{\partial r}\left(r \frac{\partial T}{\partial r}\right)
\end{aligned}
$$

where $\operatorname{Pr}$ is the Prandtl number, with the boundary conditions (4) become

$$
\begin{gathered}
v=0, u=U(x), \frac{\partial T}{\partial r}=-q_{w}(x) \text { at } r=R(x) \\
u=0, T=0 \text { as } r \rightarrow \infty
\end{gathered}
$$

In order that Eqs. (6) - (8) become similar, we take

$$
U(x)=x^{m}, q_{w}(x)=x^{(5 m-1) / 2}
$$

where $m$ is a constant. We introduce now the following similarity variables:

$$
\psi=x f(\eta), T(x)=x^{2 m} \theta(\eta)
$$


where

$$
\eta=x^{m-1} r^{2}
$$

and $\psi$ is the stream function which is defined in the usual way as $u=(1 / r) \partial \psi / \partial r$ and $v=$ $-(1 / r) \partial \psi / \partial x$. The introduction of the stream function automatically satisfies the continuity equation (6). The surfaces of constant $\eta=a$, where $a$ is a nondimensional constant and is numerically small for a slender body, corresponds to the surfaces of revolution. Setting $\eta=a$, Eq. (12) prescribes both shape and size of the body with its surface given by

$$
R(x)=a^{1 / 2} x^{(1-m) / 2}
$$

Of practical interests are pointed bodies and cylinders for which we must have, from Eq. (13), $m \leq 1$. For example, the body is a cylinder when $m=1$, a paraboloid when $m=0$, and a cone when $m=-1$. Substituting (11) and (12) into Eqs. (7) and (8), we get the following ordinary differential equations:

$$
\begin{aligned}
2\left(\eta f^{\prime \prime}\right)^{\prime}+f f^{\prime \prime}-m f^{\prime 2} & =0 \\
\frac{2}{P r}\left(\eta \theta^{\prime}\right)^{\prime}+f \theta^{\prime}-2 m f^{\prime} \theta & =0
\end{aligned}
$$

subject to the boundary conditions

$$
\begin{gathered}
f(a)=\frac{(1-m) a}{2}, f^{\prime}(a)=\frac{1}{2}, f^{\prime}(\infty) \rightarrow 0, \\
\theta^{\prime}(a)=-\frac{1}{2} a^{-1 / 2}, \theta(\infty) \rightarrow 0
\end{gathered}
$$

where primes denote differentiation with respect to $\eta$.

The physical quantities of interest are the skin friction coefficient $C_{f}$ and the wall temperature $T_{w}$ which are defined as

$$
C_{f}=\frac{\tau_{w}}{\rho \bar{U}^{2} / 2}, T_{w}=\frac{k R e^{1 / 2}\left(\bar{T}_{w}-T_{\infty}\right)}{q_{0} L}
$$

where the skin friction $\tau_{w}$ is given by

$$
\tau_{w}=\mu\left(\frac{\partial \bar{u}}{\partial \bar{r}}\right)_{\bar{r}=\bar{R}(\bar{x})}
$$

Using (5), (11), (12) and (18), we get

$$
C_{f} R e_{x}^{1 / 2}=8 a^{1 / 2} f^{\prime \prime}(a), T_{w}=x^{2 m} \theta(a)
$$

where $R e_{x}=\bar{U}(\bar{x}) \bar{x} / \nu$ is the local Reynolds number.

\section{Results and Discussion}

Generally, as mentioned in the previous section, the body is a cylinder when $m=1$, a paraboloid when $m=0$ and a cone when $m=-1$ (see Chen [7]).
However, when a solid object of any shape, such as a needle in this present problem, exhibits a rectilinear translational motion through a fluid medium, then all parts of the solid object (needle) must have the same velocity. This implies that the velocity of its surface $U$ has to be a constant and therefore, only the value $m=0$ should be considered in the present paper. However, the only exception one may think of is an elastic body. The most typical example is an elastic sheet that is being stretched, in which the sheet velocity varies along the sheet. Therefore, in the present problem, the results for $m=-1$ and $m=+1$ are not of any physical relevance since (10) is inconsistent with a solid needle. Still, these results are solutions of the mathematical problem posed, but without any physical realism. According to Eq. (13) the value of $m=0$ corresponds to a blunt-nosed needle or a paraboloid with $R(x)=a^{1 / 2} x^{1 / 2}$.

The system of decoupled ordinary differential equations (14) and (15) subject to the boundary conditions (16) has been solved numerically using an implicit finite-difference method known as the Kellerbox scheme as described in the book by Cebeci and Bradshaw [17] for $m=0$ (a blunt-nosed needle with variable heat flux) and some values of the governing parameter $a$ (in the range of $0.001 \leq a \leq 0.1$ ). We consider that the needle moves in a fluid with different Prandtl numbers, i.e. $\operatorname{Pr}$ varies in the range of $0.01 \leq \operatorname{Pr} \leq 100$. It is worth mentioning that small values of $\operatorname{Pr}(\ll 1)$ physically correspond to liquid metals, which have high thermal conductivity but low viscosity, while $\operatorname{Pr} \sim 1$ corresponds to diatomic gases including air. On the other hand, large values of $\operatorname{Pr}(\gg 1)$ correspond to high-viscosity oils and $\operatorname{Pr}=6.8$ corresponds to water at room temperature. Results are presented in 6 figures.

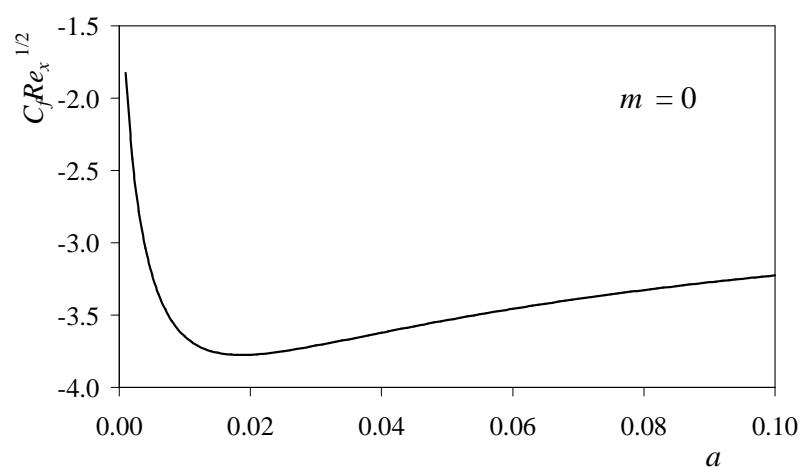

Figure 2: Variation of the skin friction coefficient with $a$ for various $\operatorname{Pr}$ when $m=0$

The variations with $a$ of the skin friction coefficient $C_{f} R e_{x}^{1 / 2}$ and the wall temperature $T_{w}$, given by expressions (19) for $m=0$, are shown in Figures 2 


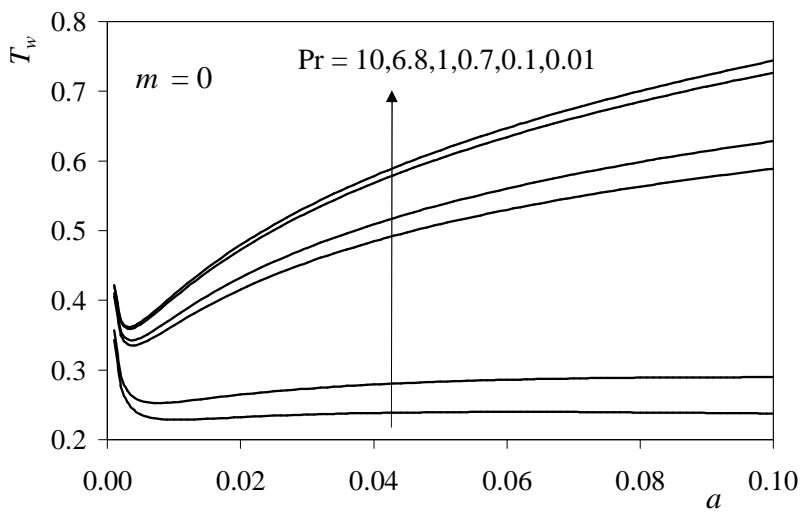

Figure 3: Variation of the wall temperature with $a$ for various $\operatorname{Pr}$ when $m=0$

and 3 for $\operatorname{Pr}=0.01,0.1,0.7,1,6.8$ and 10. Due to the decoupled boundary layer equations (14) and (15), it is seen from Figure 2 that there is only a unique skin friction coefficient for all considered values of $\mathrm{Pr}$ at different values of $a$. It can also be seen from Figure 2 that the skin friction coefficient decreases with the increase of $a$ for $0.001 \leq a \leq a_{\min }$ where $a_{\min }$ is the value of $a$ when the skin friction coefficient is minimum. Figure 2 also shows that for $a_{\min }<a \leq 0.1$, the skin friction coefficient increases with the increase of $a$. It is worth mentioning that negative sign of the skin friction coefficient in Figure 2 physically implies that the fluid produces a dragging force on the surface.

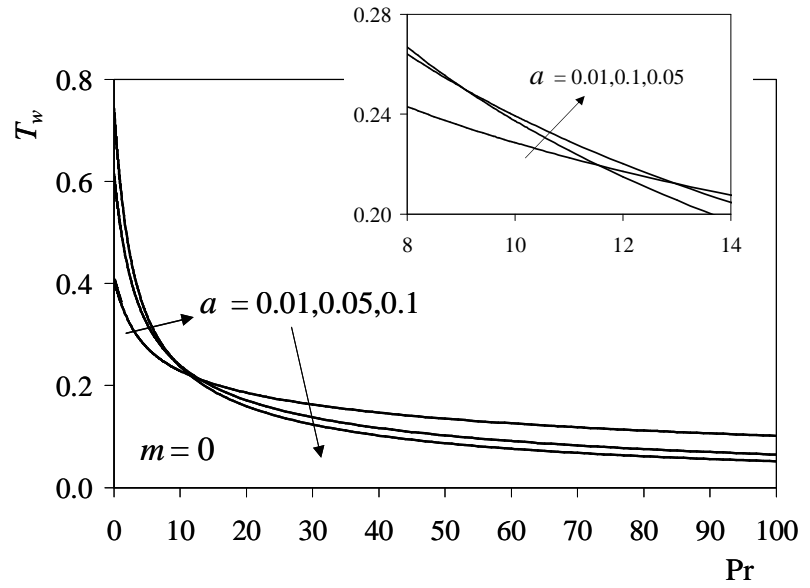

Figure 4: Variation of the wall temperature with $\mathrm{Pr}$ for various values of $a$ when $m=0$

Figures 3 and 4 show the variation with $a$ and $\mathrm{Pr}$, respectively, for the wall temperature of a bluntnosed needle with variable heat flux $(m=0)$. It can be seen from those figures that at any fixed values of $a$, the wall temperature decreases as $\operatorname{Pr}$ increases. Physically, this is because the thermal dif- fusivity in boundary layer becomes higher as $\operatorname{Pr}$ increases. Figure 3 shows that for a fixed value of $P r$, the wall temperature decreases with the increase of $a$ for $0.001 \leq a \leq a_{\min }(\operatorname{Pr})$ where $a_{\min }(\operatorname{Pr})$ is the value of $a$ when the wall temperature is minimum and depending on Pr. On the other hand, it is seen from Figure 3 that for $a_{\min }(P r)<a \leq 0.1$, the wall temperature increases when $a$ increases. Furthermore, it can be seen from Figure 4 that for $\operatorname{Pr}<\operatorname{Pr}_{i n}^{0.05,0.1}$ where $\operatorname{Pr}_{i n}^{0.05,0.1}$ is the value of $\operatorname{Pr}$ when the curves $a=0.05$ and $a=0.1$ intersect, the wall temperature increases with the increase of $a$. On the other hand, Figure 4 also shows that the wall temperature decreases with the increase of $a$ when $\operatorname{Pr}>\operatorname{Pr}_{\text {in }}^{0.01,0.05}$ where $\operatorname{Pr}_{i n}^{0.01,0.05}$ is the value of $\operatorname{Pr}$ when the curves $a=0.01$ and $a=0.05$ intersect.

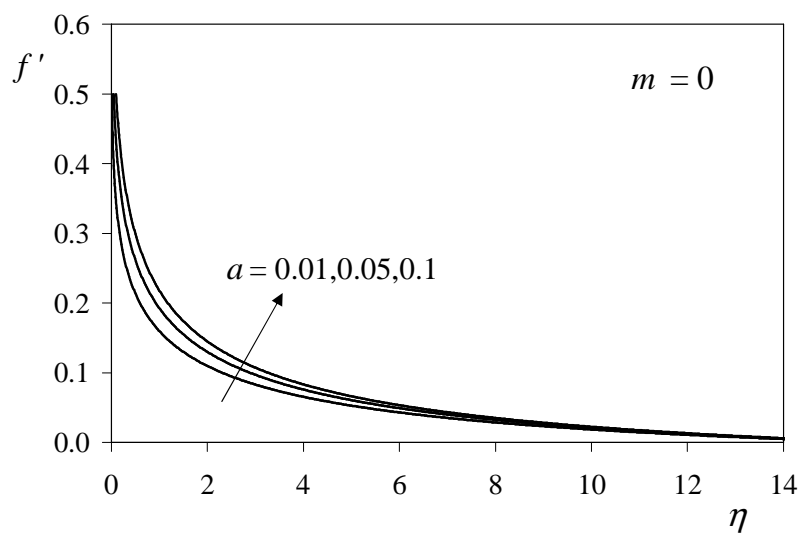

Figure 5: Velocity profiles for various values of $a$ with $m=0$

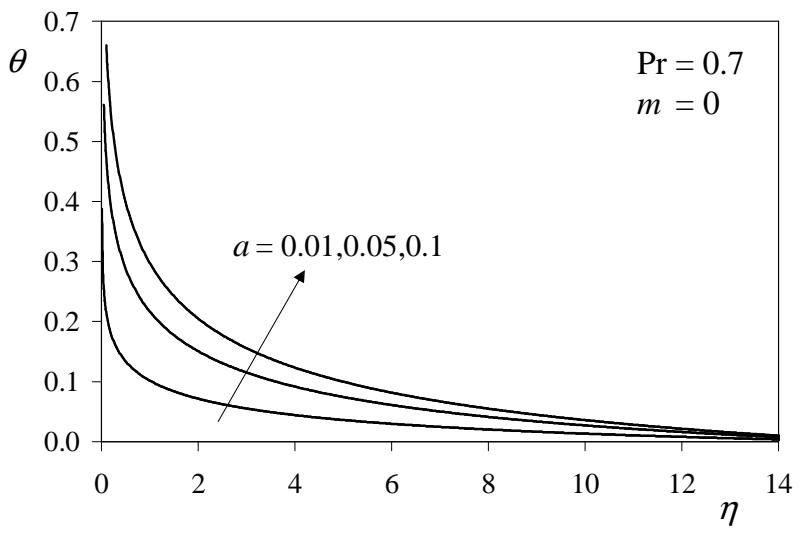

Figure 6: Temperature profiles for various values of $a$ with $\operatorname{Pr}=0.7$ and $m=0$

The axial velocity profiles $f^{\prime}(\eta)$ and the nondimensional temperature profiles $\theta(\eta)$ for a bluntnosed needle with variable heat flux $(m=0)$ are plot- 
ted versus $\eta$ in Figures 5 and 6, respectively, for three needle sizes, namely $a=0.01,0.05$ and 0.1 . Figure 5 shows that at any fixed value of $a$, there is only a unique velocity profile for all values of $\mathrm{Pr}$. It is seen from Figures 5 and 6 that the velocity and thermal boundary layer thicknesses increase with the increase of the needle size $a$. An inspection of these figures clearly shows that the thinner the needle, the smaller is the value of $\eta$ for the free stream conditions to be attained, i.e. the boundary layer thickness decreases with the decreasing values of $a$. It can also be clearly seen from Figure 5 that $f^{\prime \prime}(\eta) \rightarrow 0$ as $\eta \rightarrow \infty$, i.e. the shear stress vanishes outside the momentum boundary layer.

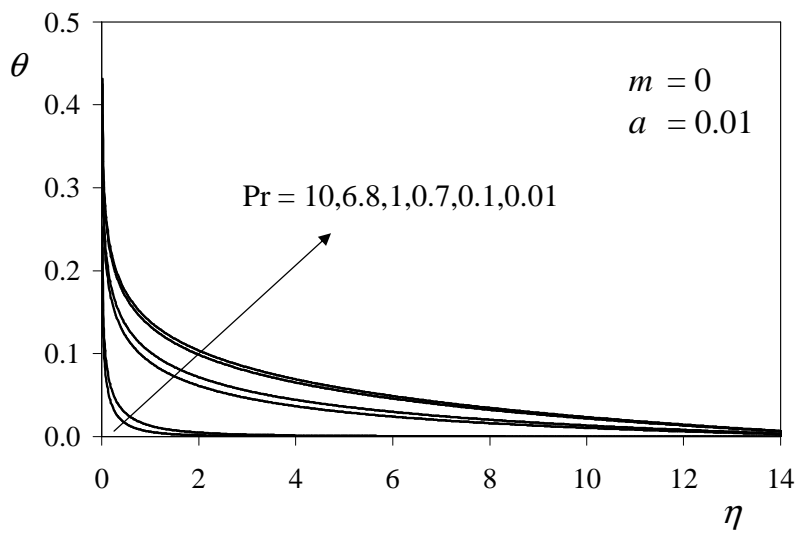

Figure 7: Temperature profiles for various $\operatorname{Pr}$ with $m=0$ and $a=0.01$

Figure 7 displays the non-dimensional temperature profile $\theta(\eta)$ for a blunt-nosed needle with variable heat flux $(m=0)$ for various values of $\mathrm{Pr}$ $(P r=0.01,0.1,0.7,1,6.8,10)$ and $a=0.01$. It is shown that the temperature profile decreases as $\mathrm{Pr}$ increases. It is also shown that the thermal boundary layer thickness decreases with an increase in $\mathrm{Pr}$. Physically, this is because, as $\operatorname{Pr}$ increases, the thermal diffusivity decreases. This leads to the decrease of the energy transfer ability that reduces the thermal boundary layer.

\section{Conclusions}

The problem of steady laminar forced convection boundary layer flows of an incompressible viscous fluid over a moving thin needle in an ambient fluid is studied. Calculations are carried out for a blunt-nosed needle with variable heat flux ( $m=0)$, which moves in a fluid with a wide range of the Prandtl numbers $(0.01 \leq \operatorname{Pr} \leq 100)$. The numerical results are also obtained for various values of the dimensionless parameters, which include the Prandtl number $\mathrm{Pr}$ and the parameter $a$ representing the needle size. The results show that the shape and the size of the needles have strong effects on the velocity and the thermal characteristics of the problem. Generally, it can be concluded that the wall temperature and the temperature profiles are significantly influenced by the considered parameters. However, the Prandtl number has no effect on the local skin friction coefficient and the velocity profiles due to the decoupled boundary layer equations.

Acknowledgements: The authors gratefully acknowledge the financial support received in the form of a research grant (Research University Grant Scheme (RUGS)) from the Universiti Putra Malaysia and Engineering Mathematics Group, Universiti Kebangsaan Malaysia (Project No.: UKM-OUP-BTT25/2007). The first author also acknowledge Universiti Sains Malaysia and Ministry of Higher Education, Malaysia, for the financial support received throughout the course of this study.

\section{References:}

[1] E.-M. Sparrow and J.-P. Abraham, Universal solutions for the streamwise variation of the temperature of a moving sheet in the presence of a moving fluid, Int. J. Heat Mass Transf. 48, 2005, pp. 3047-3056.

[2] J.-P. Abraham and E.-M. Sparrow, Friction drag resulting from the simultaneous imposed motions of a freestream and its bounding surface, Int. J. Heat Fluid Flow 26, 2005, pp. 289-295.

[3] K. Sadeghy and M. Sharifi, Local similarity solution for the flow of a "second-grade" viscoelastic fluid above a moving plate, Int. J. NonLinear Mech. 39, 2004, pp. 1265-1273.

[4] J.-L.-S. Chen and T.-N. Smith, Forced convection heat transfer from nonisothermal thin needles, J. Heat Transf. 100, 1978, pp. 358-362.

[5] J.-P. Narain and M.-S. Uberoi, Combined forced and free-convection heat transfer from vertical thin needles in a uniform stream, Phys. Fluids 15, 1972, pp. 1879-1882.

[6] J.-P. Narain and M.-S. Uberoi, Combined forced and free-convection over thin needles, Int. J. Heat Mass Transf. 16, 1973, pp. 1505-1511.

[7] J.-L.-S. Chen, Mixed convection flow about slender bodies of revolution, J. Heat Transf. 109, 1987, pp. 1033-1036.

[8] S.-L. Lee, T.-S. Chen and B.-F. Armaly, Mixed convection along vertical cylinders and needles with uniform surface heat flux, J. Heat Transf. 109, 1987, pp. 711-716. 
[9] S. Ahmad, N.-M. Arifin, R. Nazar and I. Pop, Mixed convection boundary layer flow along vertical thin needles: Assisting and opposing flows, Int. Comm. Heat Mass Transf. DOI:10.1016/j.icheatmasstransfer.2007.07 .005 (Published online on 10th August 2007).

[10] C.-Y. Wang, Mixed convection on a vertical needle with heated tip, Phys. Fluids A 2, 1990, pp. 622-625.

[11] M. Agarwal, R.-P. Chhabra and V. Eswaran, Laminar momentum and thermal boundary layers of power-law fluids over a slender cylinder, Chem. Engng. Sci. 57, 2002, pp. 1331-1341.

[12] R.-S.-R. Gorla, Unsteady viscous flow in the vicinity of an axisymmetric stagnation point on a circular cylinder, Int. J. Engng. Sci. 17, 1979, pp. 87-93.

[13] R.-S.-R. Gorla, Boundary layer flow of a micropolar fluid in the vicinity of an axisymmetric stagnation point on a cylinder, Int. J. Engng. Sci. 28, 1990, pp. 145-152.

[14] R.-S.-R. Gorla, Mixed convection in an axisymmetric stagnation flow on a vertical cylinder, Acta Mech. 99, 1993, pp. 113-123.

[15] S. Ahmad, N.-M. Arifin, R. Nazar and I. Pop, Mixed convection boundary layer flow along vertical moving thin needles with variable heat flux, Heat Mass Transf. DOI 10.1007/S00231007-0263-6 (Published online on 12th April 2007).

[16] L.-L. Lee, Boundary layer over a thin needle, Phys. Fluids 10, 1967, pp. 820-822.

[17] T. Cebeci and P. Bradshaw, Physical and Computational Aspects of Convective Heat Transfer, Springer, New York 1988. 